\title{
Dépôt par ablation laser de guides d'onde d'alumine pure et dopée en europium. Propriétés structurales et optiques
}

\author{
A. Pillonnet, C. Garapon, C. Champeaux ${ }^{*}$ C. Bovier**, R. Brenier**, L. Lou, \\ A. Catherinot* B. Jacquier et J. Mugnier \\ Laboratoire de Physico-Chimie des Matériaux Luminescents, UMR 5620 du CNRS, \\ Université Claude Bernard Lyon 1, 43 boulevard du 11 novembre 1918, 69622 Villeurbanne \\ cedex, France \\ * Laboratoire de Matériaux Céramiques et Traitements de Surface, URA 320 du CNRS, \\ Université de Limoges, 123 avenue Albert Thomas, 87060 Limoges cedex, France \\ ** Département de Physique des Matériaux, UMR 5596 du CNRS, Université Claude Bernard \\ Lyon 1, 43 boulevard du 11 novembre 1918, 69622 Villeurbanne cedex, France
}

\begin{abstract}
Résumé : Des guides d'ondes $\mathrm{d}^{\prime} \mathrm{Al}_{2} \mathrm{O}_{3}$ pure et dopée en europium ont été élaborés par ablation laser. Leurs caractéristiques structurales, leurs aptitudes au guidage optique, et leurs propriétés de fluorescence ont été étudiées en relation avec la pression d'oxygène au cours du dépôt. Les films sont stoechiométriques, amorphes avec l'amorce d'une cristallisation (structure $\gamma$ ) et présentent des indices de réfraction élevés, la pression d'oxygène influant à la fois sur l'indice du film, la vitesse de croissance et la valence de l'ion dopant.
\end{abstract}

Les guides d'onde optiques dopés par des ions fluorescents présentent des applications potentielles importantes en optique intégrée (amplification optique, effet laser...) [1]. En outre ils peuvent donner lieu à des études fondamentales, quant à l'effet du confinement de l'énergie lumineuse sur les propriétés de fluorescence. De plus, il est possible d'étudier la structure particulière de matériaux en films minces, grâce à l'utilisation de la lumière guidée lors des études spectroscopiques et au rôle de sonde structurale des ions fluorescents. La méthode de dépôt par ablation laser, d'utilisation récente dans le domaine des guides d'onde optiques, a déjà montré sa capacité à produire des films denses et exempts de défauts susceptibles de gêner la propagation de la lumière [2]. Ces possibilités sont illustrées ici par le cas de films d'alumine pure ou dopée en europium dont les caractéristiques structurales, l'aptitude au guidage optique et les propriétés de fluorescence ont été étudiées en relation avec la pression d'oxygène lors du dépôt.

Les films sont obtenus par ablation par un laser $\mathrm{KrF}(\lambda=248 \mathrm{~nm}, \mathrm{f}=10 \mathrm{~Hz})$ de cibles de poudres compactées et recuites. Tous les dépôts sont réalisés sur des substrats de silice $\left(\mathrm{SiO}_{2} / \mathrm{Si}\right.$ ou $\left.\mathrm{SiO}_{2} \mathrm{Herasil}^{(\mathbb{8})}\right)$, dont la température est de $790^{\circ} \mathrm{C}$. La fluence du laser est $3 \mathrm{~J} / \mathrm{cm}^{2}$ et la distance cible-substrat de $4 \mathrm{~cm}$ et la durée de dépôt est de 45 minutes. Nous avons étudié l'influence de la pression d'oxygène au cours du dépôt (gamme $10^{-6}-0,1$ mbar) et de la nature des cibles élaborées à partir de deux types de poudres : soit l'oxyde $\alpha-\mathrm{Al}_{2} \mathrm{O}_{3}$ (CERAC $4 \mathrm{~N}$ ), éventuellement mélangé intimement à $\mathrm{Eu}_{2} \mathrm{O}_{3}$, soit des poudres réalisées par voie solgel $\mathrm{d}^{\prime} \mathrm{Al}_{2} \mathrm{O}_{3}$ pure ou dopée par $1 \% \mathrm{Eu}^{3+}$ (lors de l'élaboration du sol), dont la structure dépend de la température de recuit.

Les films d'alumine obtenus sont transparents, exempts de craquelures et de gouttelettes, la meilleure qualité étant atteinte pour les films préparés à partir de cibles sol-gel. Les mesures de R.B.S. (Rutherford Backscattering Spectroscopy) montrent que les dépôts $\mathrm{d}^{\prime} \mathrm{Al}_{2} \mathrm{O}_{3}$ sont stoechiométriques avec un dopage en europium égal à celui des cibles et homogène dans l'épaisseur.

D'après les spectres de diffraction des rayons $X$ en configuration $\theta-2 \theta$, les films sont amorphes avec un début de cristallisation dans la phase $\gamma$ de l'alumine [3]. La spectroscopie Raman en configuration guidée [4] permet également de mettre en évidence un début d'ordre. Une étude de structure par diffraction de rayons $\mathrm{X}$ rasants est en cours afin de préciser le rôle de la température du substrat et celui de la pression d'oxygène sur la cristallisation.

L'évolution de l'indice de réfraction et de l'épaisseur des films, mesurés en spectroscopie des lignes noires [5] à $\lambda=632,8 \mathrm{~nm}$, est reportée èn fonction de la pression d'oxygène sur la figure 1 . L'accroissement de l'énergie cinétique des particules, lorsque la pression diminue [6], favorise la croissance du film ainsi que sa densification. De plus, ces films sont optiquement guidants, les pertes de propagation, déterminées par l'atténuation de la lumière diffusée en surface [7], étant de $4 \pm 0,5 \mathrm{~dB} / \mathrm{cm}$. 

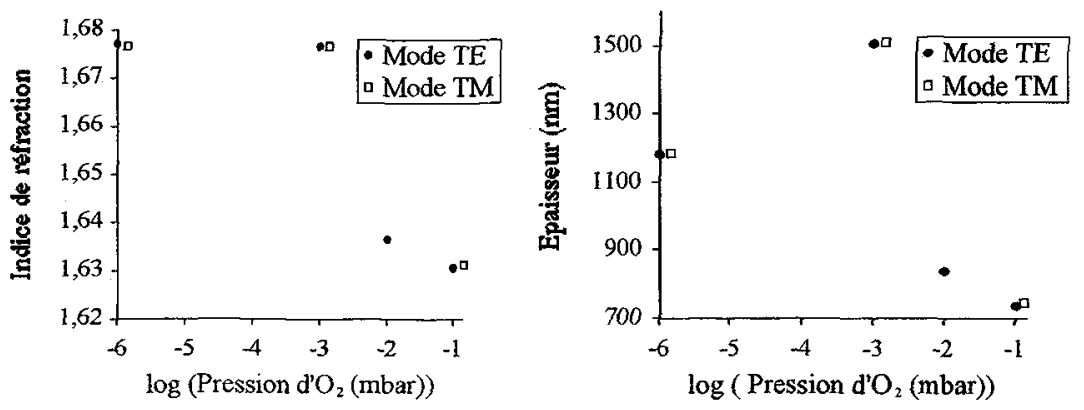

Figure 1 : Indice de réfraction à $\lambda=632,8 \mathrm{~nm}$ et épaisseur des guides d' $\mathrm{Al}_{2} \mathrm{O}_{3}$ en fonction de la pression d'oxygène

Enfin, les spectres de fluorescence des films dopés en europium ont été enregistrés sous excitation en incidence rasante par un laser à colorant pompé par un laser excimère $\mathrm{XeCl}$ à $\lambda=470 \mathrm{~nm}$ (Fig 2). Les films élaborés sous $0,1 \mathrm{mbar}$ d'oxygène présentent un spectre de fluorescence caractéristique de l'ion trivalent $\mathrm{Eu}^{3+}$, constitué des transitions du niveau ${ }^{5} \mathrm{D}_{0}$ vers les multiplets ${ }^{7} \mathrm{~F}_{\mathrm{J}}, \mathrm{J}=0$ à 4 . Les films déposés à $10^{-6} \mathrm{mbar}$ montrent le spectre de l'ion $\mathrm{Eu}^{2+}, \mathrm{c}^{\prime}$ est-à-dire la bande large $4 \mathrm{f}^{6}-5 \mathrm{~d} \rightarrow 4 \mathrm{f}^{7}$. La valence trois de l'europium est donc conservée en présence d'oxygène lors du dépôt. Elle est aussi rétablie dans les films élaborés sous vide après un recuit sous oxygène. La largeur importante des raies de fluorescence indique, pour les ions $\mathrm{Eu}^{3+}$, un environnement désordonné qui dépend des conditions d'élaboration.
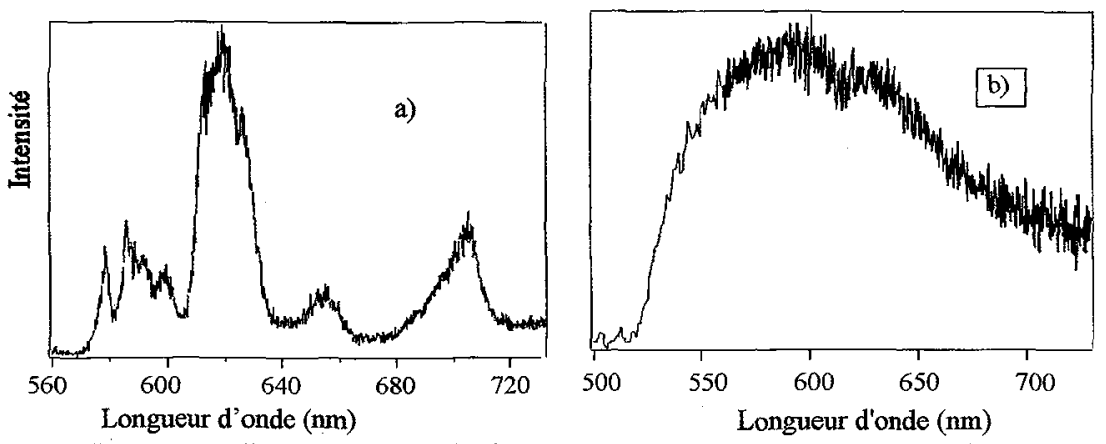

Figure 2 : Spectres de fluorescence d'un film $\mathrm{d}^{\prime} \mathrm{Al}_{2} \mathrm{O}_{3}$ dopée en europium élaboré (a) sous 0,1 mbar d'oxygène et (b) à $10^{-6} \mathrm{mbar}$.

L'ablation laser permet de réaliser des guides d'onde d'alumine pure et dopée en europium, de bonne qualité optique, et présentant des indices de réfraction, pouvant atteindre 1,713 , élevés par rapport à ceux obtenus par d'autres méthodes de dépôts. La pression d'oxygène joue un rôle important sur la vitesse de croissance, l'indice de réfraction et la valence de l'ion dopant, mais n'influe pas sur la stoechiométrie des couches d'alumine.

\section{Références}

1. C.L. Bonner, A.A. Anderson, R.W. Eason, D.P. Shepherd, D.S. Gill, C. Grivas, N. Vainos, Opt. Lett. 22, 988 (1997)

2. M. Jelinek, J. Lancok, J. Sonsky, J. Oswald, M. Simeckova, L. Jastrabik, V. Studnicka, C. Grivas, P. Hribek Czechoslovak, J. Phys. 48, 577 (1998)

3. Y. Repelin, E. Husson, Mat. Res. Bull. 25, $611(1990)$

4. C. Urlacher, J. Mugnier J. Raman Spectrosc. 27, 785 (1996)

5. M. Ulrich, R. Torge, Appl. Opt. 12, 2, 2901 (1973)

6. C. Girault, D. Damiani, J. Aubreton, A. Catherinot, Appl. Phys. Lett. 54(24), 2035 (1989)

7. M.D. Himel, U.J. Gibson, Appl. Opt. 25, 23, 4413 (1986) 